Note: This is a draft of a paper being submitted for publication. Contents of this paper should not be quoted nor referred to without permission of the author(s).

[To be published in the Proceedings of the Materials Research Society, Symposium M]

\title{
HOPPING MODEL FOR THE NON-DEBYE DIELECTRIC RESPONSE IN IONIC CRYSTALS
}

\author{
J. C. Wang and J. B. Bates
}
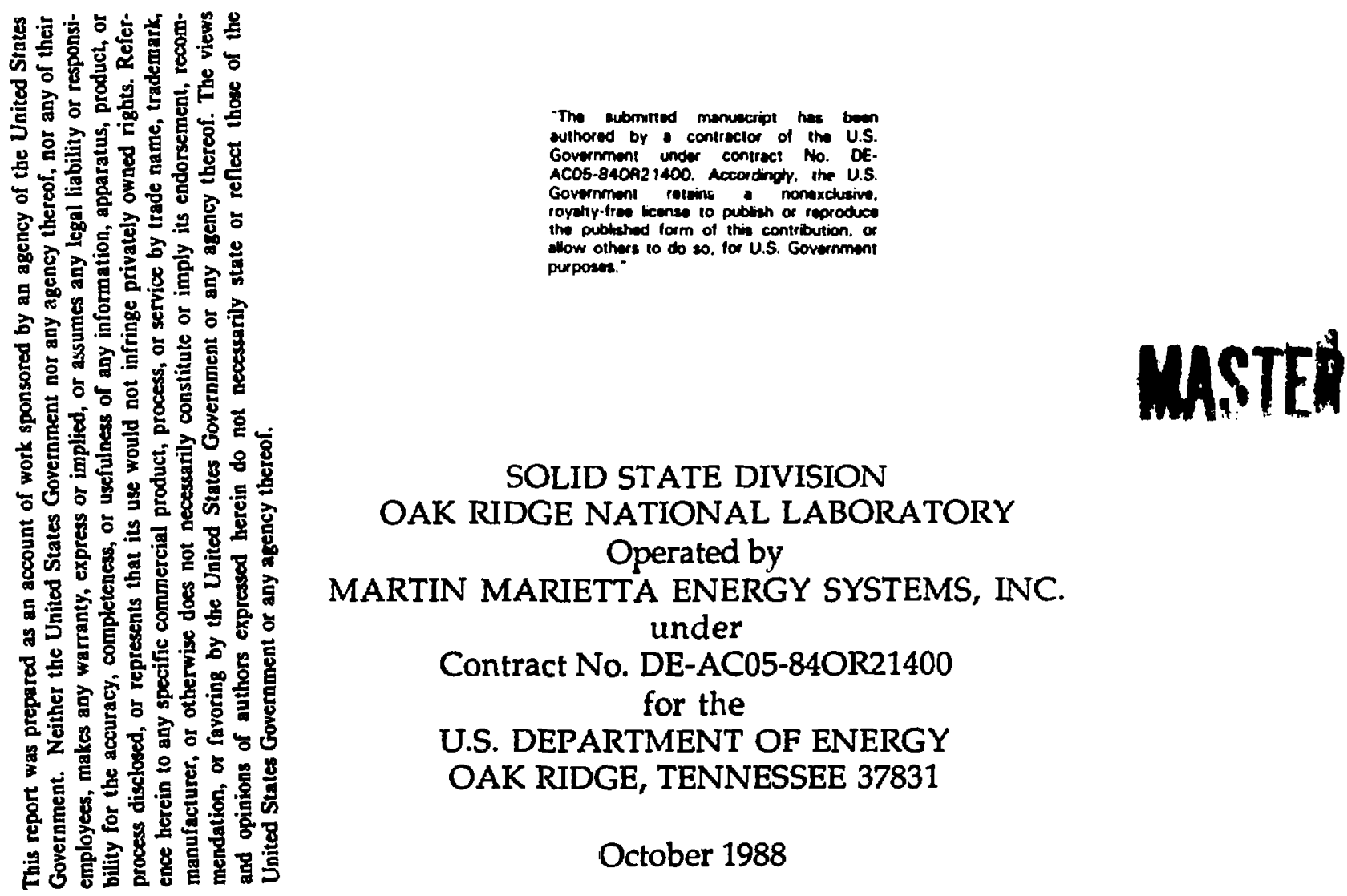
SOLID STATE DIVISION
OAK RIDGE NATIONAL LABORATORY
Operated by

MARTIN MARIETTA ENERGY SYSTEMS, INC.

under

Contract No. DE-AC05-84OR21400

for the

U.S. DEPARTMENT OF ENERGY

OAK RIDGE, TENNESSEE 37831

October 1988 


\title{
HOPPING MODEL FOR THE NON-DEBYE DIELECTRIC RESPONSE IN IONIC CRYSTALS*
}

\author{
J. C. WANG and J. B. BATES
}

Solid State Division, Oak Ridge National Laboratory, Oak Ridge, TN 37831-6030

\section{ABSTRACT}

A model based on ion hopping in potential double-wells is proposed to explain the non-Debye dielectric response in solids. Relying on some assumptions, an attempt is made to remove the "average" nature of previous diffusion theories. This results in a distribution of activation energies, $G(E)$. which decays exponentially on both sides of some given value $E_{0}$. It is shown that (a) the existence of a dielectric loss peak is a result of the decay of $G(E)$ for $E>E_{0}$, (b) the constant-phase-angle behavior above the loss peak is associated with the decay of $G(E)$ for $E<E_{0}$, and (c) $G(E)$ can produce all the main features of the empirical Havriliak-Negami function. An interesting property of this $G(E)$ is that it broadens with increasing temperature, consistent with many experimental observations.

\section{INTRODUCTION}

The bulk dielectric response of many materials shows a loss peak,[1] the existence of which can be qualitatively understood from a version of the Debye model: Charged particles associated with fixed centers, such as $\mathrm{O}^{2-}$ vacancies in stabilized zirconia, are each assumed to hop back and forth in a potential doublewell. According to Vineyard's theory of rate processes,[2] the transition rate has the thermally activated form with a single activation energy. This energy is equal to the difference between the minimum potential energy of the crystal when a hopping particle is at the saddle point and that when it is at the bottom of a well. Under a constant electric field, there are more particles in one half of the wells than the other half, and so the material has a constant polarization. When the electric field is removed at $t=0$, the polarization decays exponentially with a time constant

$$
\tau=\tau_{\mathrm{o}} \mathrm{e}^{\mathrm{E} / \mathrm{kT}}
$$

where $\mathrm{E}$ is the activation energy, $\tau_{0}$ is a characteristic constant of the material, and $\mathrm{k}$ is the Boltzmann constant. This kind of relaxation gives a complex Debye dielectric function:[3]

$$
\varepsilon(\omega)-\varepsilon_{\infty}=\frac{\varepsilon_{0}-\varepsilon_{\infty}}{1+j \omega \tau}
$$

where $\varepsilon_{0}$ and $\varepsilon_{\infty}$ are the low and high frequency dielectric constant, respectively, and $\mathrm{j}=\sqrt{-1}$. The imaginary part of $\varepsilon(\omega)$, as can be seen from Fig. 1, has a peak. However, many materials show a non-Debye dielectric response[1] characterized by a much broader loss peak and a constant-phase-angle (CPA) behavior at frequencies above the loss peak defined by $\operatorname{Re}\left[\varepsilon(\omega)-\varepsilon_{\infty}\right] / \operatorname{Im}\left[\varepsilon(\omega)-\varepsilon_{\infty}\right]=$ constant. The non-Debye form can be represented very well by an empirical function proposed by Havriliak and Negami (H-N):[4]

$$
\varepsilon(\omega)-\varepsilon_{\infty}=\frac{\varepsilon_{0}-\varepsilon_{\infty}}{\left[1+(j \omega \tau)^{1-\alpha}\right]^{\beta}}
$$


where $\tau^{\prime}$ is a constant, $0 \leq \alpha<1$, and $0<\beta \leq 1$. This dielectric function reduces to the Debye and other empirical functions in special cases: (a) Debye:[5] $\alpha=0$ and $\beta=1$, (b) Cole-Cole:[6] $\beta=1$, and (c) Davidson-Cole:[7] $\alpha=0$. The broad loss peak as well as the CPA behavior above the loss peak of the IH-N function is shown in Fig. 1 ; it can be considered as a superposition of Debye functions, Eq. (1), with some distribution of activation energies $G(E):[8]$

$$
\varepsilon(\omega)-\varepsilon_{\infty}=\int \frac{\varepsilon_{0}-\varepsilon_{\infty}}{1+j \omega \tau_{0} e^{E / k T}} G(E) d E
$$

The purpose of this work is to present a hopping model that gives a reasonable $G(E)$ and $\varepsilon(\omega)$.

Fig. 1. Comparison of the Debye and the $\mathrm{H}-\mathrm{N}$ dielectric function. The latter, with values of parameters estimated from Ref. 16 for zirconia, has a much broader loss peak and a CPA behavior above the loss peak.

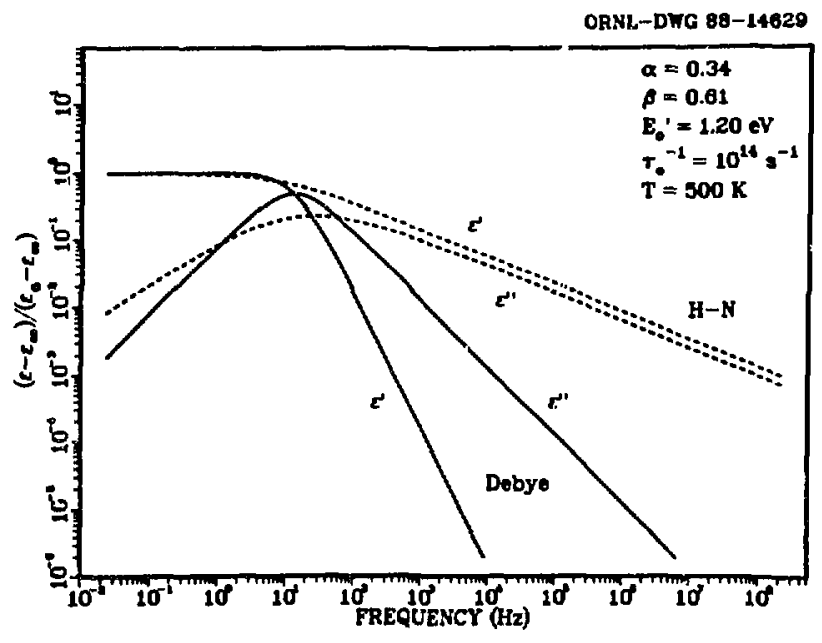

\section{GENERALIZATION OF THE DEBYE MODEL}

The fact that experimental results indicate a $G(E)$ instead of a single activation energy suggests that Vineyard's theory may need modification. One argument for doing so is that the theory gives an "average jump rate"[2] while the dielectric response function exhibits effects of all transition rates. Other diffusion theories have a similar nature;[9-12] for example, Wert uses a mean potential energy to obtain the activation energy for diffusion $[9,10]$, and Rice postulates the existence of a minimum potential configuration and considers all others negligible.[11,12] In this work, we generalize the Debye model by removing the "average" nature of these diffusion theories.

According to Vineyard's theory,[2] when the jumping particle is at the saddle point, the crystal may assume any of the various possible configurations. The probability of occurrence of a configuration depends on its potential energy in the form of Boltzmann's factor. Here we group those configurations having the same potential energy together and assume that the probability for the potential energy to be $E_{0}+V_{2}$ is given by

$$
P_{2}\left(V_{2}\right)=(b / k T) e^{-b V_{2} / k T}
$$

where $E_{0}$ is the potential energy at $T=0$ and the distribution function has been normalized. When $b=1$, Eq. (5) is the Boltzmann distribution.

Next we consider a particle that has just made a successful jump. Let the potential energy of the crystal be $V_{1}$ when the particle was near the bottom of the well before the jump. Using an argument similar to that for writing down Eq. (5), 
we assume that the probability function for $V_{1}$ is given by

$$
P_{1}\left(V_{1}\right)=(a / k T) e^{-a V_{1} / k T}
$$

Here, in this case, we have chosen the potential energy of the crystal to be zero at $T=0$. The activation energy for the jon jump is given by

$$
E=E_{0}+V_{2}-V_{1}
$$

For all the successful ion jumps, the probability function for the activation energy to be $E$ can be obtained from Egs. (5)-(7):

$$
G(E)=a b(k T)^{-2} \iint \delta\left(E-E_{0}-V_{2}+V_{1}\right) e^{-\left(b V_{2}+a V_{1}\right) / k T} d V_{1} d V_{2}
$$

Carrying out first the integration over $V_{1}\left(V_{2}\right)$ if $E<E_{0}\left(E>E_{0}\right)$ and then the second integration from 0 to $\infty$,

$$
G(F)= \begin{cases}\frac{a b}{(a+b) k T} e^{-a\left(E_{0}-E\right) / k T} & \text { for } E \leq E_{0} \\ \frac{a b}{(a+b) k T} e^{-b\left(E-E_{0}\right) / k T} & \text { for } E \geq E_{0}\end{cases}
$$

Notice that this $G(E)$ decays exponentially on both sides of $E_{0}$. Macdonald[13-15] has demoristrated that double exponential G(E)'s can yield dielectric responses very close to the empirical expressions such as the Cole-Cole,[6] Davidson-Cole,[7] and $\mathrm{H}-\mathrm{N}$ function. However, while Macdonald assumes the $G(E)$ 's to be temperatureindependent,[14,15] Eq. (9) specifies a definite form of temperature dependence.

\section{COMPARISON WITH THE H-N FUNCTION}

It is interesting to compare $G(E)$ of our model, Eq. (9), with the $G(E)$ corresponding to the empirical $\mathrm{H}-\mathrm{N}$ function which can represent experimental resuits very well. The analytical formula of the distribution of relaxation times for the $\mathrm{H}-\mathrm{N}$ function is given by: $[4,8]$

$$
g(\ln \tau)=\frac{(\tau / \tau)^{\beta(1-\alpha)} \sin \beta \theta}{\pi\left[(\tau / \tau)^{2(1-\alpha)}+2(\tau / \tau)^{1-\alpha} \cos \pi(1-\alpha)+1\right]^{\beta / 2}} .
$$

where $\tau^{\prime}$ is a constant and

$$
\theta=\tan ^{-1}\left\{\sin \pi(1-\alpha) /\left[\left(\tau / \tau^{\prime}\right)^{1-\alpha}+\cos \pi(1-\alpha)\right]\right\}
$$

From Eq. (1) and with

$$
\tau=\tau_{0} \mathrm{e}^{\mathrm{E}_{\mathrm{o}} \cdot / \mathrm{kT}}
$$

$\mathrm{G}(\mathrm{E})$ of the $\mathrm{H}-\mathrm{N}$ function can be calculated from Eqs. (10)-(12). At the low and high energy limit, it can be shown that 


$$
G_{H-N}(E)- \begin{cases}e^{-\beta(1-\alpha)\left(E_{0}-E\right) / k T} & \text { for } E<<E_{0} \\ e^{-(1-\alpha)\left(E-E_{0}\right) / k T} & \text { for } E>>E_{0^{\circ}}\end{cases}
$$

This means that $G(E)$ for the $\mathrm{H}-\mathrm{N}$ function also decays exponentially on both sides of some energy $E_{0}$ : similar to $G(E)$ of our model. Comparing Eçs. (13) and (9), one finds that $a$ and $b$ are related to $\alpha$ and $\beta$ by

and

$$
a=\beta(1-\alpha),
$$

$$
\mathrm{b}=(1-\alpha)
$$

The H-N function has been used in the equivalent circuits for analyzing the bulk ac response of $\mathrm{ZrO}_{2}$.[16] The results are encouragingly good. In Fig. 2 we show a typical $G(E)$ for the $H-N$ function obtained from fitting the experimental data. The exponential decay can be seen from the linear semi-logarithmic graphs on both sides of $E_{o}^{\prime}$. To compare this $G(E)$ with that of our model, we use Eqs. (14) and (15) to determine $a$ and $b$ from $\alpha$ and $\beta$, and choose $E_{0}$ to be the peak energy of the $G(E)$ for the $\mathrm{H}-\mathrm{N}$ function. This peak energy can be obtained numerically and is found to be slightly different from $E_{0}$ ' defined in Eq. (12). As can be seen from Fig. 2, G(E) determined in this way has all the main features of that of the empirical $\mathrm{H}-\mathrm{N}$ function. We have also compared the dielectric response calculated with these two $G(E)^{\prime} s$. The graphs are almost indistinguishable.

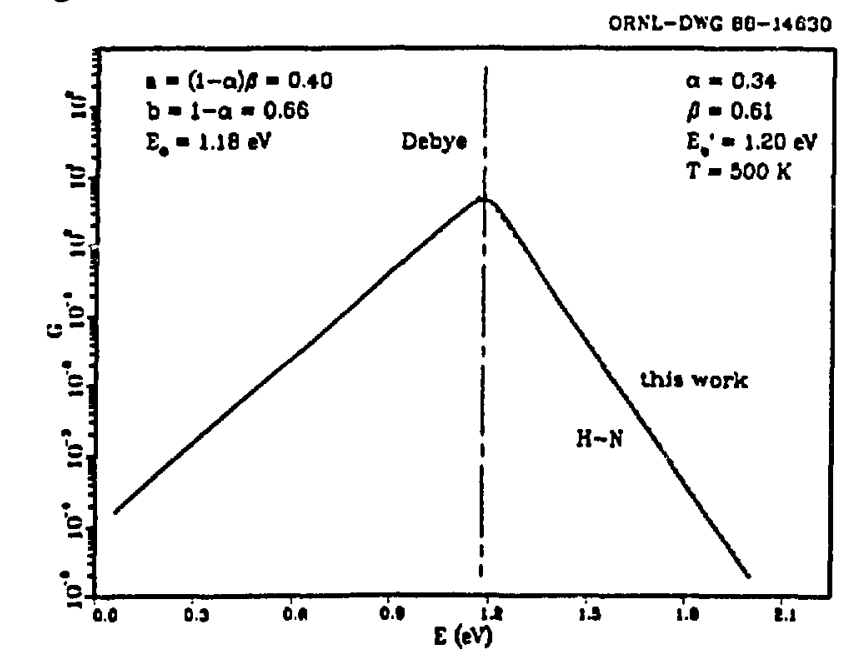

Fig. 2. Comparison of $G(E)$ 's. $E_{0}$ for the present model, obtained numerically, is taken to be the peak position of the $\mathrm{H}-\mathrm{N} \mathrm{G}(\mathrm{E})$ and has a slightly different value from $E_{0}$. The $G(E)$ for the Debye function is shown as a delta function.

The log-log graph of the imaginary part of the H-N function (showing the loss peak) has the asymptotic form $[4,17]$

$$
\begin{aligned}
\varepsilon^{\prime \prime}(\omega) \sim \omega^{m} \text { for } \omega<<1 / \tau^{\prime} \\
-\omega^{-n} \text { for } \omega>>1 / \tau^{\prime}
\end{aligned}
$$

where $m$ and $n$ are related to $\alpha$ and $\beta$ by

and

$$
m=1-\alpha,
$$

$$
n=\beta(1-\alpha)
$$


From Eqs. (14), (15), (17), and (18), these frequency exponents are related to the parameters $a$ and $b$ in the present model by

and

$$
\mathrm{m}=\mathrm{b} \text {, }
$$

$$
\mathbf{n}=\mathbf{a} \text {. }
$$

\section{CPA BEHAVIOR AND LOSS PEAK}

As can be seen from Fig. 1, the H-N function has a CPA behavior for frequencies above the loss peak. Since higher frequencies correspond to smaller activation energies [Eq. (1)], this behavior can be associated with the exponential decay of $G_{H-N}(E)$ in Eq. (13). In our model, Eq. (9), the exponential decay of $G(E)$ below $E_{0}$ is, therefore, responsible for the CPA behavior. Because $E$ is positive, this decay can span at most from $E=E_{0}$ to $E=0$; and it is possible chat for a crystal with small $E_{0}$, the CPA behavior is limited only to some frequency range. Eq. (9) to

If we extrapolate the exponential form of $G(E)$ for $E<E_{0}$ to $E>E_{0}$, i.e., changing

$$
G(E)=\frac{a b}{(a+b) k T} e^{-a\left(E_{0}-E\right) / k T} \text { for all } E \text {, }
$$

then $G(E)$ will increase exponentially with $E$ and the CPA behavior will extend to very low frequencies. The decrease of $G(E)$ above $E_{0}$ in $E q$. (9), therefore, is responsible for the termination of the CPA behavior at low frequencies and is responsible for the appearance of the loss peak.

\section{TEMPERATURE DEPENDENCE OF G(E)}

As can be seen from Eq. (9), the present model predicts a temperature dependence of $G(E)$; it broadens with increasing $T$ as illustrated in Fig. 3. To see the experimental evidence for this, we first notice that in many cases, the $\log -\log$ graphs of complex dielectric function vs. frequency at different temperatures have almost

Fig. 3. Broadening of $G(E)$ with increasing temperature predicted by Eq. (9) of the present model. The values of parameters were estimated from Ref. 16 for zirconia.

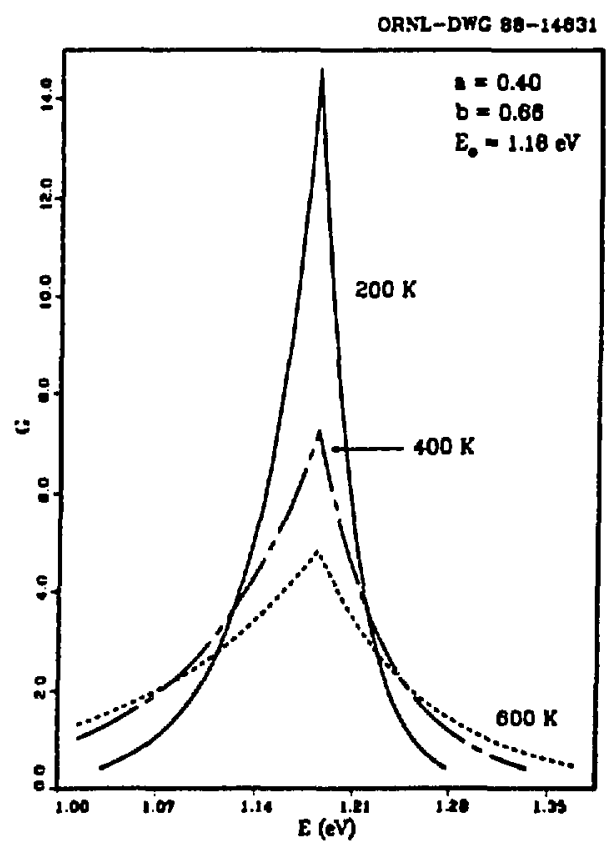


an identical shape and can be superimposed by simply shifting the origin of the coordinates.[1] In Jonscher's words, "very many sets of experimental data may be satisfactorily normalized into reasonably single-valued master curves, thereby proving that the spectral shape of the loss characteristics remains invariant with temperature."[18] This implies that the two frequency exponents of the loss peak, $m$ and $\mathrm{n}$ in Eq. (16), are independent of T. From Egs. (19) and (20), one finds that $a$ and $b$ for these materials also are independent of $\mathrm{T}$. From Eq. (9), one can, therefore, argue that the $G(E)$ 's of these materials should broaden with increasing temperature as shown in Fig. 3. We have used the $\mathrm{H}-\mathrm{N}$ function in the equivalent circuits for analyzing the bulk ac response of $\mathrm{ZrO}_{2} \cdot[16]$ The results also lead to constant frequency exponents $m$ and $n$, suggesting a broadening of $G(E)$ with $T$ in that material.

\section{DISCUSSION}

There are several possible origins for a distribution of activation energies, for example, different local environments and interaction among hopping particles. The $G(E)$ considered in this work is believed to arise from the existence of various crystal configurations due to thermal agitation; therefore, it should exist even when all hopping particles have the same environment. This implies that the Debye dielectric response, which corresponds to a single activation energy, should not be commonly observed in solids, and indeed it is not.[1]

At present, the parameters $a$.nd $b$ used in Egs. (5) and (6) cannot be justified with certainty. One possibility is that they result from grouping configurations having the same potential energy. Another possibility is the existence of "effective temperatures," $T / a$ and $T / b$, when the hopping particle is at the bottom of a well and at the saddle point, respectively.

\footnotetext{
"Research sponsored by the Division of Materials Science, U.S. Department of Energy under contract DE-AC05-84OR- 21400 with Martin Marietta Energy Systems, Inc.
}

\section{REFERENCES}

1. A. K. Jonscher, Dielectric Relaxation in Solids, (Chelsea Dielectrics Press, London, 1983).

2. G. H. Vineyard, J. Phys. Chem. Solids 3, 121 (1957).

3. J. C. Anderson, Dielectrics, (Reinhold Publishing Corp., New York, 1964), p. 67.

4. 5. Havriliak and S. Negami, Polymer 8, 161 (1967).

5. P. Debye, Polar Molecules, (Dover, New York, 1929).

6. K. S. Cole and R. H. Cole, J. Chem. Phys. 9, 341 (1941).

7. D. W. Davidson and R. H. Cole, J. Chem. Phys. 19, 1484 (1951).

8. C. J. F. Bottcher and P. Bordewijk, Theory of Electric Polarization, (Elsevier, Amsterdam, 1978).

9. C. A. Wert, Phys. Rev. 79, 601 (1950).

10. A. B. Lidiard, in Encyclopedia of Physics (Handbuch der Physik), edited by $\mathrm{S}$. Flugge (Springer, Berlin, 1957), Vol. XX: Electrical Conductivity II, pp. 246-349.

11. S. A. Rice, Phys. Rev. 112, 804 (1958).

12. H. R. Glyde, Rev. Mod. Phys. 39, 373 (1967).

13. J. R. Macdonald, J. Appl. Phys. 58, 1971 (1985).

14. J. R. Macdonald, J. Appl. Phys. 61, 700 (1987).

15. J. R. Macdonald, J. Appl. Pliys. 62, R51 (1987).

16. J. B. Bates and J. C. Wang, Solid State lonics (in press).

17. S. Yagihara, R. Nozaki, S. Takeishi, and S. Mashimo, J. Chem. Phys. 79, 2419 (1983).

18. p. 105 of Ref. 1. 